\title{
Determinación de la presencia de residuos antibióticos betalactámicos en los productos lácteos expendidos en la ciudad de Cajamarca
}

\section{Determination of the presence of beta-lactam antibiotic residues in dairy products expended in Cajamarca city}

Gutiérrez-Arce, Felipe 1[0000-0002-8547-6897]; Rojas-Vásquez, Zulema ${ }^{2[0000-}$ 0003-0290-2203]; Quispe-Rodríguez, Brando 3[0000-0001-8693-2914]; Quito-

Vásquez, Fanny 3[0000-0003-1285-6562]; Llasac-Rojas, Milagros 3[0000-0002-29137014]; Terán-Piña, Julio 1[0000-0001-9438-0486] y Gutiérrez-Arce, Walter ${ }^{4[0000-}$

0002-5498-4362]

${ }^{1}$ Universidad Nacional de San Martín, Tarapoto, Perú

${ }^{2}$ Práctica privada

${ }^{3}$ Universidad Privada Antonio Guillermo Urrelo, Cajamarca, Perú

${ }^{4}$ Universidad Nacional de Cajamarca, Perú fbgutierrezeunsm.edu.pe

Resumen. El trabajo tuvo como objetivo determinar la presencia de residuos antibióticos betalactámicos en el queso mantecoso expendidos en Cajamarca, 2019. Para ello, durante el mes de agosto, se tomó una muestra semanal en cada uno de los seis puntos de expendios seleccionados. Se eligió a aquellas tiendas que vendían más de $30 \mathrm{~kg}$ de queso mantecoso por día. También se compararon los resultados de los análisis con los Límites Máximos del Residuo (LMR) de los antibióticos del grupo de los betalactámicos, considerados por los Codex Alimentarius (para amoxicilina, ampicilina, y penicilina) y por el Reglamento de la Unión Europea (para cefalexina), para determinar la calidad del producto. Se obtuvo que el antibiótico con mayor presencia fue la penicilina; incluso, en uno de los puntos de muestreo, rebasa hasta en casi un $300 \%(11.13 \mu \mathrm{g} / \mathrm{kg})$, el LMR permitido por el Codex Alimentarius $(4 \mu \mathrm{g} / \mathrm{kg})$. Y otro punto de muestreo, también mostró cantidades de residuos de penicilina en sus productos $(3.52 \mu \mathrm{g} / \mathrm{kg})$ muy cercanos a los permitidos. Concluyendo que, aproximadamente, 1 de cada 6 puntos de ventas de queso mantecoso sobrepasa el LMR de penicilina, producto de uso veterinario y de efecto residual prolongado, que incluso, como ya estamos demostrando, permanece en los productos de transformación, como el queso.

Citar como: GutiérrezArce, F., Rojas-Vásquez, Z., Quispe-Rodríguez, B., Quito-Vásquez, F., LlacsaRojas, M., Terán-Piña, J. \& Gutiérrez-Arce, Walter. (2021). Determinación de la presencia de residuos antibióticos betalactámicos en los productos lácteos expendidos en la ciudad de Cajamarca. Revista de Veterinaria y Zootecnia Amazónica, 1(1), 15-21. https://doi.org/10.51252/re vza.v1i1.135

Recibido: 06/04/2021 Aceptado: 09/06/2021 Publicado: 28/07/2021
Palabras clave: antibiótico betalactámico, Cajamarca, penicilina, queso mantecoso

\begin{abstract}
The objective of the work was to determine the presence of betalactam antibiotic residues in the buttery cheese sold in Cajamarca, 2019. For this, during the month of August, a weekly sample was taken in each of the six selected outlets. chose those stores that sold more than $30 \mathrm{~kg}$ of buttery cheese per day. The results of the analyses were also compared with the Maximum Residue Limits (MRLs) of the antibiotics of the beta-lactam group, considered by the Codex Alimentarius (for amoxicillin, ampicillin, and penicillin) and by the European Union Regulation (for cephalexin), to determine the quality of the product. It was found that the antibiotic with the greatest presence was penicillin; even, at one of the sampling points, it exceeds up to almost $300 \%(11.13 \mu \mathrm{g} / \mathrm{kg})$, the MRL allowed by the Codex Alimentarius $(4 \mu \mathrm{g} / \mathrm{kg}$ ). And another sampling point also showed amounts of penicillin residues in their products $(3.52 \mu \mathrm{g} / \mathrm{kg})$ very close to those allowed. Concluding that approximately 1 in 6 sales points of buttery cheese exceeds the MRL for penicillin, a product for veterinary use and with a long residual effect, which even, as we are already demonstrating, remains in transformation products, such as cheese.
\end{abstract}

Keywords: antibiotics beta-lactam, Cajamarca, penicilin, buttery chesse 


\section{$1 \quad$ Introducción}

El mercado peruano de los lácteos, actualmente, se cimienta en dos actores que son fundamentales para la "supervivencia" de esta actividad. (1) menciona que una de ellas es la cadena industrial constituida por industrias transnacionales (Nestlé y Gloria, principalmente) y sus respectivos proveedores, y, el otro actor es la cadena artesanal que incorpora a numerosos pequeños productores no especializados, y que venden lo sobrante, a través de circuitos de comercialización a menudo informales, después de auto consumir una parte de su producción, después de transformarlos o en forma de leche cruda. Cajamarca, Arequipa, Lima y la Libertad son las principales proveedoras de la primera cadena (industrial).

Cajamarca, como región, posee tres grandes corredores económicos: 1. Económico de la zona sur (Cajamarca, San Marcos, Cajabamba, San Pablo, San Miguel, Contumazá y Celendín). Aquí resaltamos la presencia de Gloria y Nestlé, y de los mayores centros de producción de derivados lácteos, que se encuentran en Cajamarca y Baños del Inca. En el caso de Nestlé y Gloria, son 300000 litros de leche acopiados diariamente. 2. Económico del centro (Bambamarca, Chugur y Hualgayoc), donde se da la mayor producción de queso fresco y tipo suizo, y expendido a la costa, especialmente a las ciudades de Trujillo, Chiclayo y Lima. 3. Económico del norte (Chota y Cutervo), con mayor producción de queso mantecoso, andino y tipo suizo, que se venden, especialmente, en las ciudades de la costa norte y Lima. Y la producción de estos productos sigue en aumento (2).

Además, se sabe que es una de las principales ciudades que elaboran productos lácteos en el Perú, con aproximadamente 8000 toneladas producidos hasta el año 2015 entre queso fresco, maduro yogurt, manjar blanco, etc. (3). Sumado a lo anterior, tenemos que en Cajamarca el consumo de productos lácteos como el queso es una característica de arraigo cultura, lo que traería un gran problema a la salud si es que no se ofrecen productos de calidad e inocuos. El consumo per cápita de queso fresco en la ciudad es de $1.74 \mathrm{~kg}$, muy cerca al consumo del país, con $2.44 \mathrm{~kg}(4)$.

Si bien es cierto, por lo mencionado previamente, es difícil no guardar expectativas sobre las oportunidades que esta actividad podría generar para la economía de nuestro país y también de nuestra región. Sin embargo, también exige un mayor control en la cadena productiva, velando porque todos los factores que la conforman guarden los niveles permitidos y poder cumplir los estándares exigidos en los mercados internacionales, pero principalmente, evitar poner en riesgo la salud del consumidor, dado que como menciona (5), si no somos responsables en el uso de fármacos veterinarios, podría generar una consecuencias graves en la salud del consumidor, como son: sensibilidad, resistencia, alergias, cambios en la flora intestinal. 
Por lo dicho anteriormente, no son pocos los trabajos que se han realizado, teniendo en cuenta la importancia del tema, como ya quedó establecido. Tanto a nivel internacional como a nivel nacional, la preocupación manifestada ha sido y es, poder caracterizar los productos lácteos que consumimos $(6)(7)(8)(9)(10)(11)(12)$. Ahora, la gran mayoría de los trabajos se han concentrado en la leche, como objetivo de estudio, sin embargo, el queso es un producto que aún se debe estudiar. Sin embargo, como nos dejan ver estos estudios, se viene incumpliendo los Codex Alimentarius, dado que absolutamente, todos los trabajos coinciden en que existe presencia de antibiótico y en varios casos, superan los LMR permitidos.

Razón de ello es que nos proponemos el siguiente problema: ¿Las cantidades de residuos de antibióticos betalactámicos de los quesos mantecosos expendidos en la ciudad de Cajamarca, exceden los LMR que exige la Normativa (Codex Alimentarius o Reglamento de la Unión Europea)?

Consecuentemente, determinamos la presencia de residuos antibióticos betalactámicos en los productos lácteos (queso mantecoso) expendidos en la ciudad de Cajamarca, y compararlos con los exigidos por la Normativa respectiva.

La hipótesis que pondremos a prueba es la siguiente: los quesos mantecosos que se comercializan en Cajamarca presentan cantidades de residuos de antibióticos betalactámicos que no exceden los LMR que exige la Normativa (Codex Alimentarius o Reglamento de la Unión Europea).

\section{Materiales y Métodos}

Es un estudio de tipo descriptivo, de corte transversal. La metodología utilizada consistió en obtener $200 \mathrm{~g}$ de queso mantecoso de cada uno de los 6 puntos de expendio elegidos aleatoriamente.

La recolección de la muestra se realizó de julio a agosto de 2019 (período de estiaje), en 6 puntos distintos de la ciudad. Se tomó una muestra de cada lugar de manera semanal, los días miércoles. Éstas fueron colectadas en bolsas estériles rotulados con el nombre de la empresa o persona que expende (en código), dirección de donde se expende y el lugar de procedencia. Luego fueron depositadas en una heladera portátil con gel congelado y trasladadas al laboratorio para su análisis respectivo de acuerdo con la técnica de cada determinación. Finalmente, las muestras fueron analizadas en el Laboratorio Asdelab SAC, ubicado en la ciudad de Lima.

La población fue la totalidad de los puntos de expendio de queso mantecoso en la zona centro de la ciudad de Cajamarca. Está ubicada en la zona nor-andina del Perú. Su relieve es bastante accidentado. Está constituido por zonas de costa, sierra y selva. Cuenta con una extensión 
territorial de 3331754 km2. La agricultura es la principal actividad económica de la Región, y la base productiva del distrito de Cajamarca es principalmente, la actividad agropecuaria; sin embargo, su participación en el PBI regional ha disminuido en los últimos años ante el crecimiento de la actividad minera (2).

La unidad de análisis está dada por cada uno de los seis puntos de expendio donde se obtuvieron las muestras de queso mantecoso, distribuidos en los diferentes barrios de la ciudad de Cajamarca

Para elegir los puntos se muestreo, se utilizó el criterio inclusivo de considerar sólo a aquellos que vendan por encima de los $30 \mathrm{~kg}$ de queso mantecoso por día. Consecuentemente, los que estén por debajo de esta cantidad, no fueron elegidos.

Para el procesamiento de la muestra, se tomó $1 \mathrm{~g}$ de queso y $1 \mathrm{ml}$ de Acetonitrilo (ACN) $(1 \mathrm{~g} / 1$ ml.), se agitó la muestra en vórtex durante 10 segundos. Luego, la muestra fue centrifugada por 10 minutos a $14000 \mathrm{rpm}$; se extrajo el sobrenadante y se agregó $2.5 \mathrm{ml}$. de H2O grado HPLC. Esta mezcla fue sometida a extracción en fase sólida mediante columnas ODS LC18, previamente acondicionadas con $500 \mathrm{ml}$. de metanol y $500 \mathrm{ml}$. de H2O (HPLC). Después de agregar la muestra, esta fue lavada con $1 \mathrm{ml}$. de $\mathrm{H} 2 \mathrm{O}$; se dejó secar el cartucho por 5 minutos y se eluyó la molécula con $2 \mathrm{ml}$. de metanol. Luego el eluído se evaporó y se reconstituyó con $300 \mathrm{ml}$. de fase móvil y se inyectó $100 \mathrm{ml}$. directamente al sistema cromatográfico.

El registro de la información se realizó in situ, donde se buscó la toma de datos para su posterior análisis. Los datos se procesaron mediante el análisis descriptivo y el Software Infostat (versión estudiantil) que fue utilizado para realizar cálculos de medias para las diferentes muestras obtenidas. Se realizó la comparación de medias a través de la prueba de " $t$ " de Student, para comparar la determinación de penicilina en los puntos de muestreo. También se expresaron estadígrafos de dispersión, como el desvío estándar.

Para el reporte de los resultados de los análisis de laboratorio, no se menciona el lugar de origen de cada muestra analizada, sino que cada lugar de muestreo fue reportado con una letra en específico. Así mismo, los investigadores velaron permanentemente, por el correcto manejo de los datos, cuidando de que los resultados no sean manipulados para conveniencia de algunos.

\section{Resultados y discusiones}

\subsection{Determinación de la presencia de residuos antibióticos betalactámicos y comparación con los LMR establecidos.}

La tabla 3 deja ver que el antibiótico con mayor presencia fue la penicilina. Incluso, en uno de los seis puntos de muestreo (punto 3), rebasa hasta en casi un $300 \%(11.13 \mu \mathrm{g} / \mathrm{kg}$ ), el LMR 
permitido por el Codex Alimentarius ( $4 \mu \mathrm{g} / \mathrm{kg}$ ). Cabe mencionar que el punto 1 también muestra cantidades de residuos de penicilina en sus productos $(3.52 \mu \mathrm{g} / \mathrm{kg})$ muy cercanos a los permitidos.

Tabla 1.

Comparación de los LMR establecidos por Cantidades de presencia de antibióticos betalactámicos, en los puntos muestreo (de expendio de quesos).

\begin{tabular}{rccrr}
\hline \multirow{2}{*}{$\begin{array}{c}\text { Punto de } \\
\text { muestreo }\end{array}$} & \multicolumn{4}{c}{ Promedio $(\boldsymbol{\mu g} / \mathbf{k g}) \pm$ Desvío Estándar } \\
\cline { 2 - 5 } & Aoxicilina & Ampicilina & Cefalexina & \multicolumn{1}{c}{ Penicilina } \\
\hline 1 & $0.0271 \pm 0.0000$ & $0.0057 \pm 0.0007$ & $0 \pm 0.0$ & $3.5171 \pm 3.5510$ \\
2 & $0.0127 \pm 0.0014$ & $0.0033 \pm 0.0003$ & $0 \pm 0.0$ & $1.0035 \pm 0.3251$ \\
3 & $0.0212 \pm 0.0000$ & $0.0034 \pm 0.0028$ & $0 \pm 0.0$ & $11.1291 \pm 7.6265$ \\
4 & $0.0085 \pm 0.0031$ & $0.0017 \pm 0.0019$ & $0 \pm 0.0$ & $0.4619 \pm 0.0000$ \\
5 & $0.0045 \pm 0.0018$ & $0.0036 \pm 0.0023$ & $0 \pm 0.0$ & $1.9121 \pm 1.6445$ \\
6 & $0.0058 \pm 0.0050$ & $0.0042 \pm 0.0009$ & $0.0032 \pm 0.0$ & $2.5152 \pm 2.9736$ \\
4 & 4 & $100 * *$ & 4 \\
\hline \multicolumn{5}{c}{4} \\
LMR* $(\mu \mathrm{g} / \mathrm{kg})$
\end{tabular}

Los resultados nos dejan deducir que 1 de cada 6 puntos de ventas de quesos mantecosos, sobrepasa el LMR de penicilina, producto de uso veterinario y de efecto residual prolongado, que incluso, como ya estamos demostrando, permanece en los productos de transformación, como el queso.

A manera de discusión, podemos decir que, en términos cualitativos (presencia o ausencia), los resultados coinciden con trabajos como los (8)(9)(12); dado que también en estas investigaciones, la detección de antibióticos betalactámicos, como la penicilina, fue positivo. Pero cabría mencionar que el objetivo de éstos fue detectarlos en leche fresca. Sin embargo, aunque la literatura no es tan abundante al llevar el objetivo a quesos, los trabajos hallados también coinciden con nuestros resultados, dado que para autores como (6), (7), (11) y (10), también fue positiva la presencia de penicilina en quesos de distintos tipos.

El único trabajo que nos aportó un dato cuantitativo de presencia de penicilina, fue el de (10), donde reportó cantidades de residuo en antibiótico que oscilaba entre el $3.1 \mu \mathrm{g} / \mathrm{kg}-14 \mu \mathrm{g} / \mathrm{kg}$. Nuestros resultados de presencia de residuo de penicilina, oscilaron entre $0.4619 \mu \mathrm{g} / \mathrm{kg}$ y $11.1291 \mu \mathrm{g} / \mathrm{kg}$. Por lo que podríamos decir que, en ambos estudios, se originó una preocupación por estos valores que exceden, por mucho, los LMR exigidos por el Codex Alimentario.

He ahí la importancia de este trabajo, y de trabajos que sigan la misma línea, dado que necesitamos seguir monitoreando la calidad de los alimentos que consumimos, sobre todo los productos lácteos, de los que somos uno de los principales productores a nivel nacional. Y al mismo tiempo, a partir de los resultados de esta y futuras investigaciones, se debe exigir a las 
autoridades pertinentes, un mayor control y fiscalización de estos productos, especialmente, en salvaguarda de la población.

\section{Conclusiones}

Se determinó la presencia de antibióticos betalactámicos en los quesos mantecosos expendidos en la ciudad de Cajamarca, y observamos que, a diferencia de la amoxicilina, ampicilina y cefalexina (casi imperceptibles), la penicilina fue encontrada en una cantidad importante.

Al comparar los resultados con los LMR del Codex Alimentarius, pudimos ver que, en un punto de los muestreados, se excedía el LMR (11.13 $\mu \mathrm{g} / \mathrm{kg}$ vs $4 \mu \mathrm{g} / \mathrm{kg})$.

\section{Referencias bibliográficas}

1. Aubron C. Productores andinos de queso artesanal y liberalizacion del mercado de los lacteos en el Perú. Debate Agrar [Internet]. 2006;(40-41):119-39. Disponible en: https://cepes.org.pe/wp-content/uploads/2019/03/06-aubron.pdf

2. Sánchez Urteaga LJ. Informe Económico del departamento de Cajamarca para la Zonificación ecológica y económica (2010-2011) [Internet]. Gobierno Regional de Cajamarca; 2011. Disponible en: https://zeeot.regioncajamarca.gob.pe/sites/default/files/MemSocioeconomica.pdf

3. SIEA. Anuario Estadístico Producción Agroindustrial Alimentaria 2015 [Internet]. Lima: Ministerio de Agricultura y Riego; 2017. Disponible en: https://www2.congreso.gob.pe/sicr/cendocbib/con5_uibd.nsf/FE409D9008B14DB30525 81C6005385FA/\$FILE/anuario-produccion-agroindustrial-alimentaria2015_1.pdf

4. INEI. Encuesta Nacional de Presupuestos Familiares (ENAPREF) 2009 [Internet]. 2021. Disponible en: https://www.datosabiertos.gob.pe/dataset/encuesta-nacional-depresupuestos-familiares-enapref-2009-instituto-nacional-de-estadística

5. Harvey W, Hill H. Milk: Production and Control. London L\& C, editor. 1967.

6. Solares Flores ÁV. Determinación del antibiótico Penicilina en leche de vaca y su repercusión en el rendimiento del procesamiento de queso fresco [Internet]. Universidad de San Carlos de Guatemala; 2019. Disponible en: http://www.repositorio.usac.edu.gt/id/eprint/12896

7. Magallón Carrizales KB, Torres Ramírez MA, Noa Pérez M, Pacheco Gallardo C, González Aguilar DG, Mariño Guerrero IE. Monitoreo de antibióticos en quesos industrializados y artesanales en Jalisco. e-CUCBA. 2017;4(8):25-8. doi:https://doi.org/10.32870/e-cucba.v0i8.80

8. Camacho Díaz LM, Cipriano Salazar M, Cruz Lagunas B, Gutiérrez Segura I, Hernández 
Ruiz PE, Peñaloza Cortez I, et al. Residuos de antibióticos en leche cruda comercializada en la región Tierra Caliente, de Guerrero, México. REDVET [Internet]. 2010;11(2). Disponible en: https://www.redalyc.org/pdf/636/63613118006.pdf

9. Máttar S, Calderón A, Sotelo D, Sierra M, Tordecilla G. Detección de antibióticos en leches: un problema de salud pública. Rev Salud Pública. agosto de 2009;11(4):579-90. doi:https://doi.org/10.1590/S0124-00642009000400009

10. López Aldama P, Martínez Maya JJ, Sánchez del Angel LS. Determinación de penicilina y otros inhibidores en quesos frescos de la ciudad de Oaxaca, México. Vet México [Internet]. 1997;28(3):185-8. Disponible en: https://pesquisa.bvsalud.org/portal/resource/pt/li1-227433

11. Ticona Chayña E. Validación de una prueba biológica para detectar residuos de antibióticos en queso tipo paria [Internet]. Universidad Nacional del Altiplano; 2017. Disponible en: http://repositorio.unap.edu.pe/handle/UNAP/7975

12. Llanos Cortesana GA. Determinación de residuos de antibióticos en la leche fresca que consume la población de Cajamarca, Perú. Rev Amaz Investig Aliment [Internet]. 2002;2(2):35-43. Disponible en: https://www.unapiquitos.edu.pe/pregrado/facultades/alimentarias/descargas/vol3/4.pdf

\section{Conflicto de intereses}

Los autores declaramos que no existen conflictos de interés.

\section{Contribuciones de los autores}

Felipe Gutiérrez-Arce: Director del proyecto de investigación y, por ende, encargado de las coordinaciones respectivas. Así mismo, uno de los partícipes en la redacción del artículo.

Zulema Rojas-Vásquez: Partícipe principal en la redacción del proyecto y artículo final.

Brando Quispe-Rodríguez: Coordinación de trabajo de campo y muestreo.

Fanny Quito-Vásquez: Trabajo de campo y muestreo.

Milagros Llasac-Rojas: Trabajo de campo y muestreo.

Julio Terán-Piña: Encargado de aportar información y estructurar el marco teórico.

Walter Gutiérrez-Arce: Encargado del análisis de las muestras realizadas en el laboratorio. 